\title{
Functional Validation of Intelligent Retinal Stimulator Using Microchip-embedded Smart Electrode
}

\author{
Toshihiko Noda, ${ }^{*}$ Makito Haruta, Kiyotaka Sasagawa, Takashi Tokuda, and Jun Ohta \\ Graduate School of Materials Science, Nara Institute of Science and Technology, \\ 8916-5 Takayama, Ikoma, Nara 630-0192, Japan
}

(Received June 28, 2017; accepted September 5, 2017)

Keywords: retinal prosthesis, neural stimulation, suprachoroidal-transretinal stimulation, smart electrode, CMOS microchip, implantable device

A smart electrode that has a built-in CMOS microchip was fabricated for use as a retinal stimulator used in retinal prostheses. The CMOS microchip was embedded into the core of a bullet-shaped stimulus electrode with a microcavity. Because the microchip has a multiplexer function and a stimulus current generator, sophisticated functions could be implemented by the smart electrode. An array of more than one thousand electrodes, which would restore sight in high resolution, could be realized by using this smart electrode. A proof-of-concept electrode was fabricated through the development of an assembly process. We evaluated the fabricated electrode implanted in extracted animal tissue to simulate practical use. Based on this ex vivo experiment, the fabricated electrode showed optimal performance for retinal stimulation. This result is a first step in the development of next-generation retinal prostheses, which will accomplish the restoration of high-resolution sight capable of character recognition.

\section{Introduction}

In the field of sensory prostheses, many devices have been developed for practical use in clinical medicine. Retinal prosthesis technology is attracting attention as a candidate for restoring the sight of blind patients. ${ }^{(1)}$ Certain retinal prosthesis systems have been granted CE marking and/or Food and Drug Administration (FDA) approval for chronic implantation. ${ }^{(2-7)}$ Retinal prostheses are in the start-up phase of practical use. Currently, the recovery of eyesight is restricted because of the very limited number of stimulus points compared with the number of retinal cells. Increasing the number of stimulus points is one of the issues that must be resolved to provide high-resolution visual information to patients.

There are three major methods for retinal prosthesis: epi-retinal stimulation, sub-retinal stimulation, and suprachoroidal-transretinal stimulation (STS). ${ }^{(8)}$ Figure 1 shows a schematic representation of STS. In the STS approach, an array of stimulus electrodes is implanted into an intrascleral pocket located underneath the choroid layer of the eyeball. The STS approach is safer than other approaches, because the stimulus electrodes do not touch the retina and

*Corresponding author: e-mail: t-noda@ms.naist.jp

http://dx.doi.org/10.18494/SAM.2018.1649 


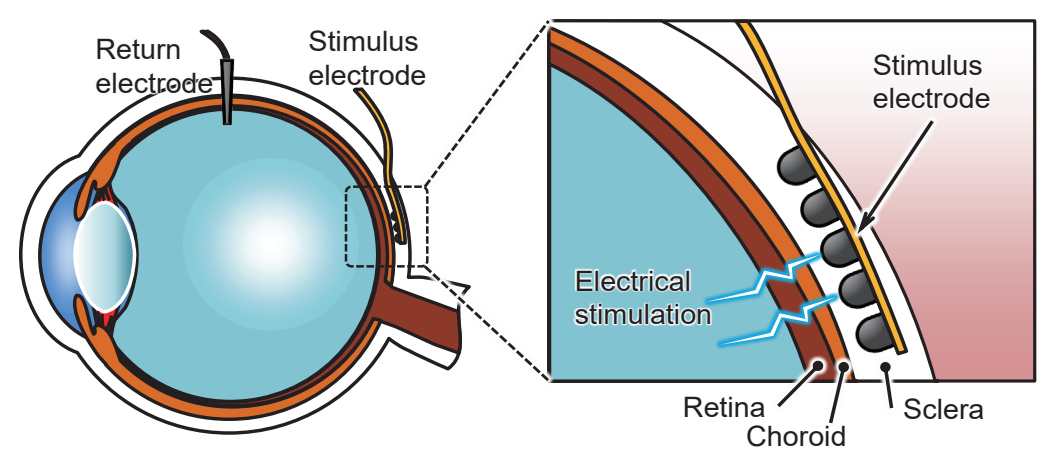

Fig. 1. (Color online) Schematic of STS.

no intraocular surgery is required. Although the gap between the stimulus electrode and the retinal cells is one advantage of STS in terms of retinal protection, this gap also causes a requirement for a high stimulus intensity for evoking the retinal cells due to the stimulus current spreading. Although the stimulus threshold varies depending on various parameters, such as electrode dimension, stimulus pulse parameters, and the implantation condition, based on our experience, a stimulus current of $100-200 \mu \mathrm{A}$ is typically required to evoke the retinal cells. ${ }^{(9,10)}$ Therefore, in the STS, large electrodes are required to provide the current (charge) density limit for safe stimulation of the retina. In the case of STS, clinical trials have been successfully performed. $^{(11)}$ A larger array of stimulus electrodes is required for the next-generation retinal stimulator. To help accomplish this, we have developed electrode arrays using smart-wiring technology combined with a CMOS microchip..$^{(9,10,12)}$ These arrays can control more than one thousand electrodes using only four wires. However, poor durability can be expected, because the CMOS microchips are exposed on a flexible substrate without a metal casing. Moreover, the architecture has an intrinsic weak point, i.e., extra space is required for CMOS microchip implementation, which prevents the high-density integration of stimulus electrodes.

To solve these problems, we propose a smart electrode device with an embedded CMOS microchip inside the electrode. Figure 2 shows a conceptual figure of the smart electrodes. Satisfactory durability can be expected because the CMOS microchip is protected by an electrode that acts as a metal casing. The proposed architecture requires no external footprint for microchip assembly; therefore, high-density arrays can be fabricated. In this study, we designed and fabricated a prototype of the smart electrode. The stimulus function of the fabricated electrode was demonstrated through an ex vivo experiment using extracted animal tissue. Whereas the concept of the smart electrode and results of its fundamental characterization were described in our previous work, ${ }^{(13)}$ detailed discussions were not provided therein. In this paper, we describe the detailed design of the key components: the microchip, flexible substrate, and stimulus electrode. The process flow of microchip separation and the assembly of the components are also explained in detail. From the results of the functional validation, the electrical properties of the fabricated electrode, which directly reflect the built-in microchip characteristics, are analyzed and discussed in detail. 


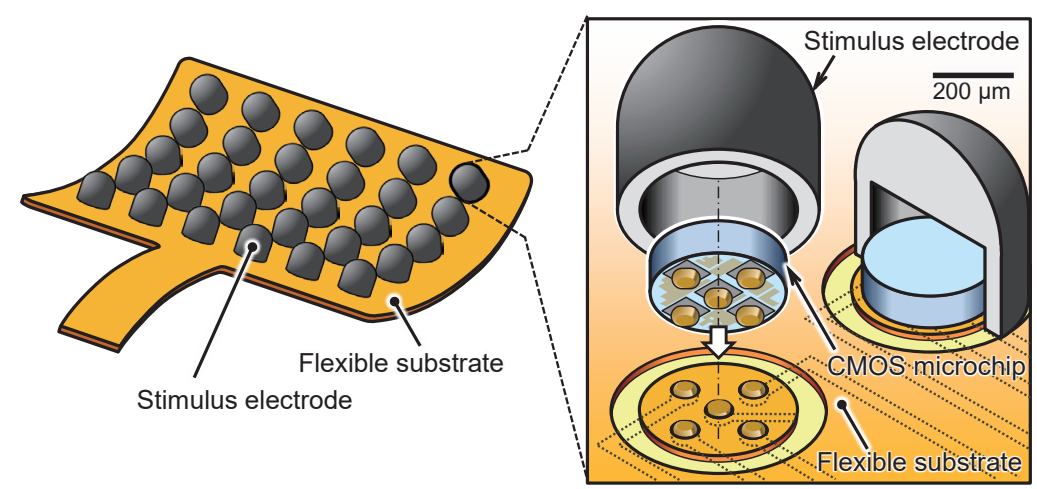

Fig. 2. (Color online) Conceptual figure of a smart electrode array. The inset shows the structure of the smart electrodes with the built-in CMOS microchips.

\section{Fabrication of the Device}

In this section, the design and fabrication processes of the smart electrode are explained in detail, after a brief explanation of its structure. As shown in Fig. 2, the bullet-like outer shape of the electrode is the same as that of an STS prototype system used in clinical trials. ${ }^{(1)}$ The electrode has a microcavity at its bottom, into which a circular CMOS microchip is embedded. Both the microchip and the electrode are mounted on a flexible substrate.

\subsection{Dedicated CMOS microchip}

A dedicated microchip was designed for the smart electrode. The design specifications are shown in Table 1. Figure 3 shows a block diagram of the circuit modules integrated into the microchip. The microchip has a multiplexer function and a stimulus pulse generator. There are five external connections: two DC power supplies, two control signals, and a stimulus output. Even in the case of a large-scale array of the smart electrode, such as more than one thousand electrodes, the microchip can be operated using four wires. A target microchip can be selected and controlled using its intrinsic chip ID number, which is up to 1024 (10 bit). The microchip was designed with $0.35 \mu \mathrm{m}$ standard CMOS technology, as shown in Fig. 4. To embed the microchip into a stimulus electrode, the microchip was designed to be circular, with a diameter of $370 \mu \mathrm{m}$.

Ten microchips were fabricated per die. Conventional dicing methods, i.e., mechanical dicing, cannot be applied to the separation of the microchips, because the outer shape of the microchips is circular. Therefore, the fabricated microchips were separated by etching the perimeter of the microchips. This separation process plays a role not only in chip separation but also in processing the outer shape of the microchip into a circle. Figure 5 shows a schematic of the process flow of the microchip separation. The initial state is as shown in Figs. 5(a-1) and 5(b-1). In the first step, as shown in Figs. 5(a-2) and 5(b-2), a Ti film, which was used as 
Table 1

Design specifications of the microchip.

\begin{tabular}{lc}
\hline Technology & $0.35 \mu \mathrm{m}$ standard CMOS process \\
Power supply voltage & DC $5.0 \mathrm{~V}$ \\
Number of input pads & $4\left(\mathrm{~V}_{\mathrm{DD}}, \mathrm{GND}\right.$, Control \#1, Control \#2) \\
Number of output pads & 1 (stimulus output) \\
Chip ID number & Maximum 1024 (10 bit) \\
Stimulus mode & Biphasic constant current pulse \\
Stimulus current intensity & Maximum $\pm 1000 \mu \mathrm{A}(50 \mu \mathrm{A}$ step) \\
Chip size & $\phi 370 \mu \mathrm{m}($ circle) \\
Chip thickness & $100 \mu \mathrm{m}$ \\
\hline
\end{tabular}

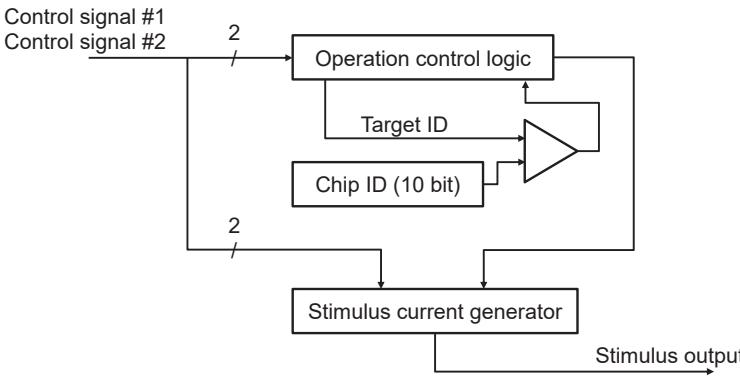

Fig. 3. Block diagram of the dedicated CMOS microchip for the smart electrode.

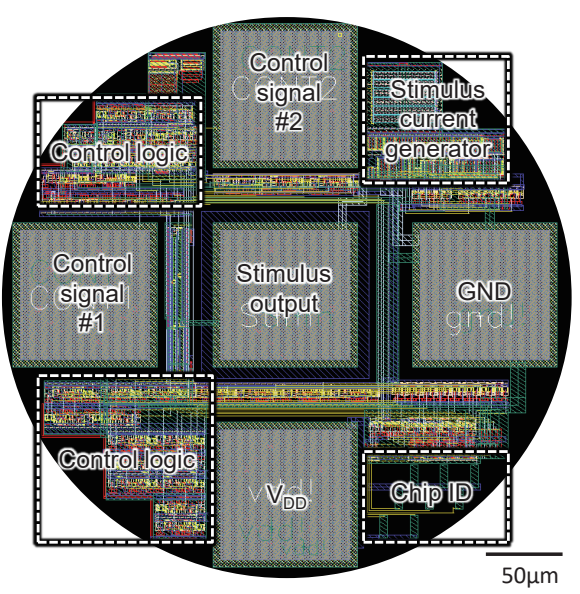

Fig. 4. (Color online) CAD layout of dedicated CMOS microchip for the smart electrode.

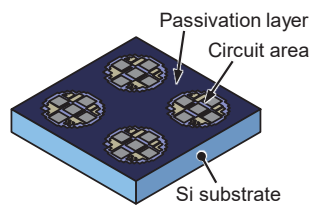

(a-1)

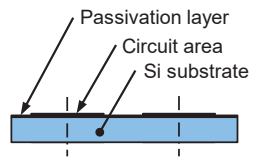

(b-1)

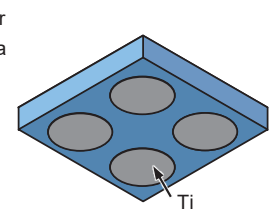

$(\mathrm{a}-2)$

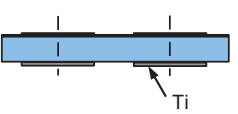

(b-2)

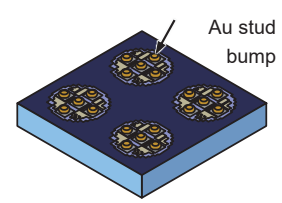

(a-3)

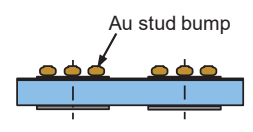

(b-3)

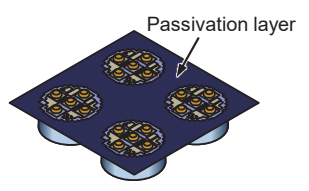

(a-4)

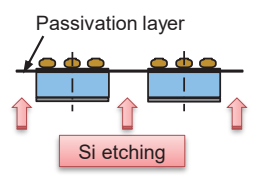

$(\mathrm{b}-4)$

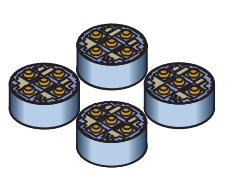

$(a-5)$

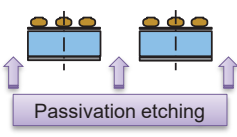

(b-5)

Fig. 5. (Color online) Process flow of microchip separation. (a-1)-(a-5) show the process flow in plan view, and (b-1)(b-5) show it in cross-sectional view.

an etching mask, was deposited and patterned on the back side of the die, i.e., the side opposite the CMOS circuit layer. Then, Au stud bumps were formed on the microchips' electrode pads, as shown in Figs. 5(a-3) and 5(b-3). Next, the Si substrate of the microchip was etched from 
the back side of the Ti mask. The Si etching was stopped, as shown in Figs. 5(a-4) and 5(b4), when the passivation layer of the microchip was exposed. Finally, as shown in Figs. 5(a5) and 5(b-5), the passivation layer was etched completely, and the microchips were separated. Simultaneously, the outer shapes of the microchips were processed into circles. Figure 6 shows a micrograph of a dedicated microchip after the chip separation process.

\subsection{Stimulus electrode}

Bullet-shaped bulk electrodes were fabricated with the dimensions shown in Fig. 7. The diameter and height of the electrode are 550 and $500 \mu \mathrm{m}$, respectively. Ti was used as the electrode material, and the electrodes were fabricated from a Ti block using a precision machining process. The tips of the electrodes were rounded to the same shape as those used in the clinical trial. ${ }^{(11,14)}$ The bottom core of the electrode was milling-processed to form a microcavity that accommodates the dedicated microchip. Because Ti has insufficient electrochemical performance for neural stimulation, the surfaces of the electrodes were coated with a 200-nm-thick Pt film.

\subsection{Flexible substrate}

A flexible substrate was designed and fabricated as shown in Fig. 8. The substrate is a conventional double-side flexible printed circuit (FPC) made of polyimide. The internal wiring of the FPC was $\mathrm{Cu}$. The electrode pads were plated with Au. One end of the 135-mm-long FPC is the stimulus head that has a $6 \times 5$ electrode array. Each electrode site of the array has five electrode pads for the CMOS microchip connection and a donut-shaped electrode pad for the

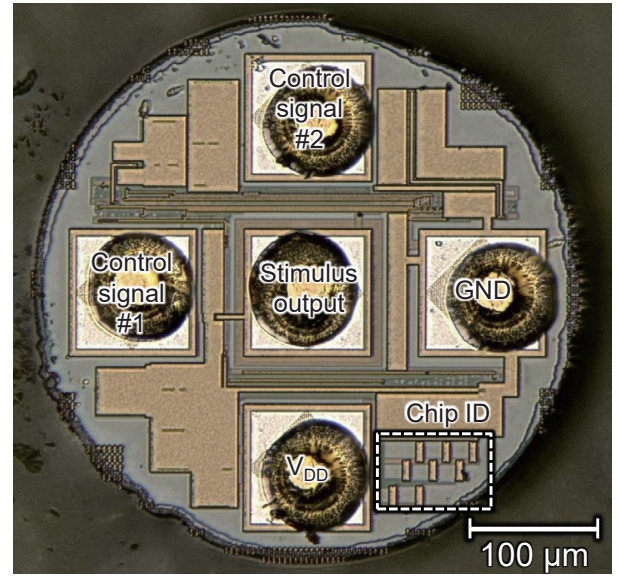

Fig. 6. (Color online) Micrograph of dedicated microchip after the chip separation process.

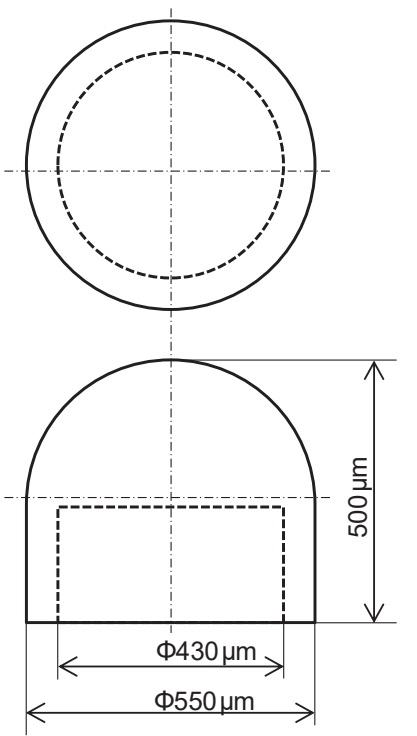

Fig. 7. Plan of stimulus electrode with a microcavity. 


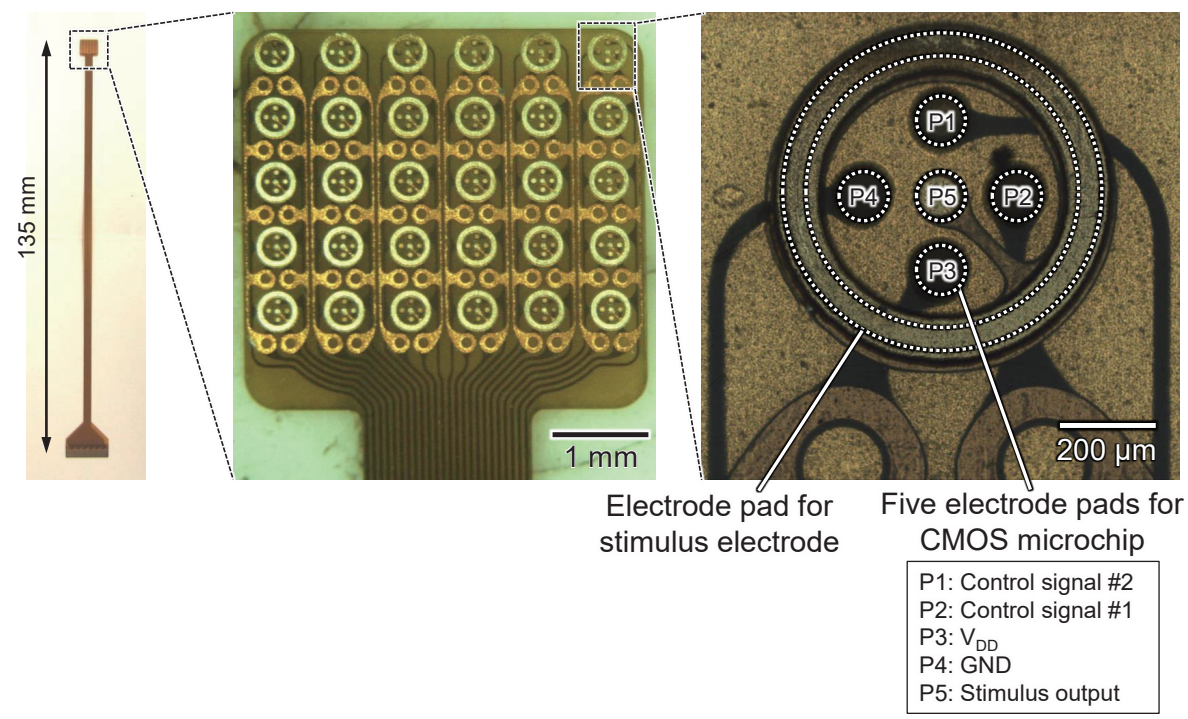

Fig. 8. (Color online) Photograph of the flexible substrate. The center inset shows the stimulus head with the 6 $\times 5$ electrode site. The enlargement on the right shows a micrograph of electrode pads for the CMOS microchip and stimulus electrode. The five electrode pads (P1-P5) correspond to the electrode pads on the CMOS microchip shown in Figs. 4 and 6. A donut-shaped pad connected to pad P5 is used for mounting the stimulus electrode.

stimulus electrode. Since the CMOS microchip has a multiplexer function, all electrodes on the stimulus head can be controlled by four bus wires. However, in this study, independent bus wirings were designed for each column to allow redundancy in the first prototype of the smart electrode array.

\subsection{Assembly of smart electrode}

In the first step of the assembly process, Au stud bumps were formed on the five electrode pads of the FPC, which were used for the CMOS microchip connections, to level the height of the electrode pads. After the pads were leveled, the separated microchip was flip-chip-bonded onto the flexible substrate, as shown in Fig. 9. Anisotropic conductive paste (TAP0402E, KYOCERA Chemical Corporation) was used in the flip-chip bonding as the conductor and reinforcing material. The bullet-shaped stimulus electrode was mounted on the flexible substrate to cover the microchip. Using SEM images of the microchip and the stimulus electrode, we show a schematic of the stimulus electrode assembly in Fig. 10. The microchip was fully embedded in the microcavity. The bottom of the stimulus electrode was connected to the donut-shaped pad on the FPC by conductive resin (EPO-TEK H20E, Epoxy Technology). Finally, the bottom perimeter of the stimulus electrode, which is the boundary between the microchip and the FPC, was made water-tight by sealing with epoxy resin. Figure 11 shows a fabricated smart electrode after the assembly process. 


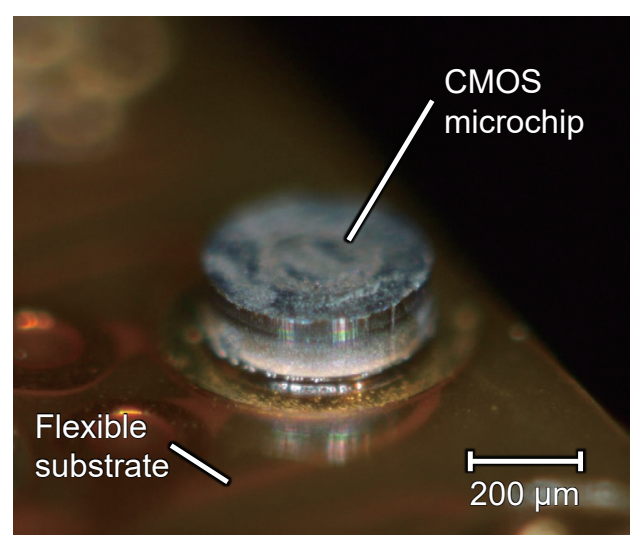

Fig. 9. (Color online) Photograph of CMOS microchip after the flip-chip bonding process.

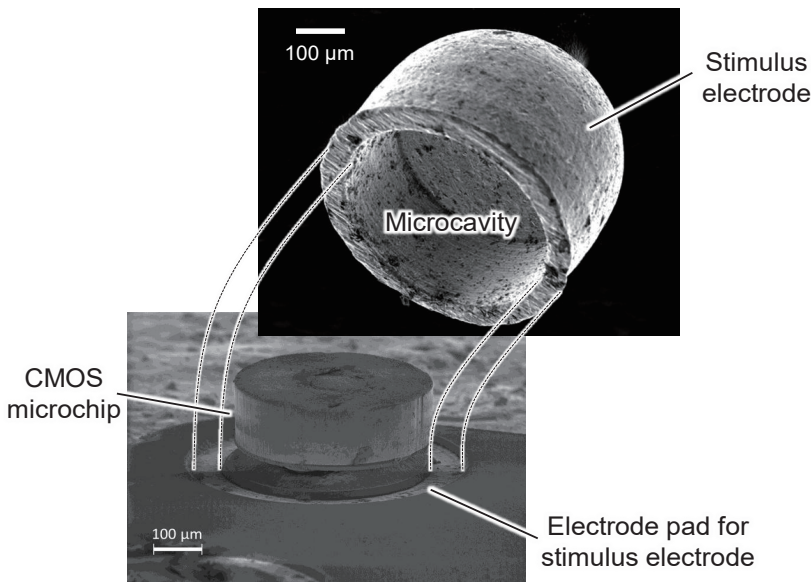

Fig. 10. Schematic of the electrode assembly.

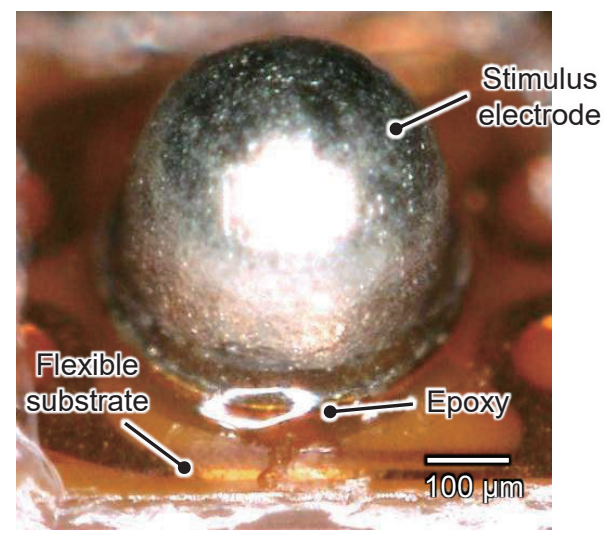

Fig. 11. (Color online) Photograph of the assembled smart electrode.

\section{Functional Validation}

\subsection{Evaluation without implantation}

In the first stage of the functional validation, the fabricated electrode was evaluated without implantation to confirm the operation of the electrode and to characterize the electrical properties. DC power and control signals were supplied to the electrode. A probe needle was contacted with the top of the fabricated electrode to measure the output current. A resistance of $500 \Omega$ was connected to the probe as a dummy load in place of biological tissue.

The output controllability was evaluated. The fabricated electrode was programed to monophasic stimulation, i.e., anodic stimulation or cathodic stimulation. The programed output intensity was varied from 50 to $1000 \mu \mathrm{A}$. The output current of each programed value was measured, as shown in Fig. 12. The output intensity can be controlled on the basis of the 


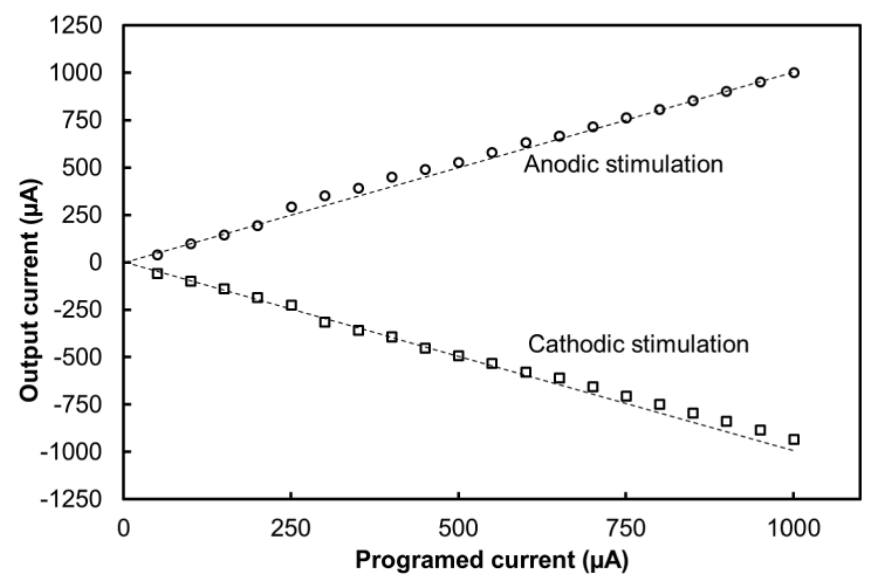

Fig. 12. Output linearity versus programed current. The polarity of the output current was positive for the source current from the electrode, and negative for the sink current to the electrode. The dashed lines show ideal output versus programed current.

programed current value. The output tolerance to the ideal value was less than $5 \%$. The output controllability of the fabricated electrode was confirmed to have sufficient accuracy for retinal stimulation.

The property of biphasic stimulation, which is necessary for the safe stimulation of the retina, was evaluated. The fabricated electrode was programed to the anodic-first biphasic stimulation mode with a stimulus intensity of $600 \mu \mathrm{A}$, pulse duration of $700 \mu \mathrm{s}$, interpulse duration of $350 \mu \mathrm{s}$, and repetition interval of $2.2 \mathrm{~ms}$. Figure 13 shows the measured waveform in the anodic-first biphasic stimulation mode. Complementary current pulses with the programed pulse parameters were successfully observed.

\subsection{Ex vivo experiment}

An ex vivo experiment using biological tissue was performed to evaluate the stimulus function. Figure 14 shows the setup of the ex vivo experiment. An extracted pig eyeball was used for the experiment. The fabricated electrode and a return electrode, which is a Pt wire 500 $\mu \mathrm{m}$ in diameter and $1 \mathrm{~cm}$ in length, were implanted into the eyeball with the STS configuration. For the electrode implantation, an intrascleral pocket was formed in the eyeball. Then the fabricated electrode was inserted into the pocket, as shown in Fig. 15. A return electrode was implanted into the side opposite the fabricated electrode. Control signals were supplied from a laptop computer via a control box. DC power was also supplied to the electrode from the control box. The fabricated electrode was programed and operated in the biphasic stimulation mode with different stimulus currents as a parameter. A current to voltage $(I-V)$ converter using an operational amplifier (TLV2372, Texas Instruments Incorporated), which was implemented in the control box, was inserted into the stimulus current path to monitor the stimulus current. Waveforms of the stimulus current were recorded using an isolated oscilloscope (GR-7000, Keyence Corporation). 


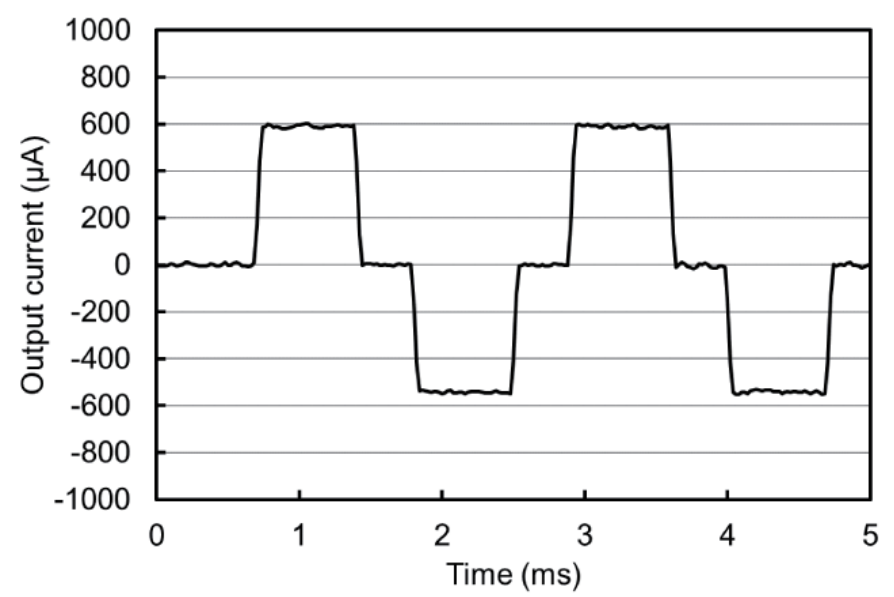

Fig. 13. Waveform of output current in anodic-first biphasic mode operation.

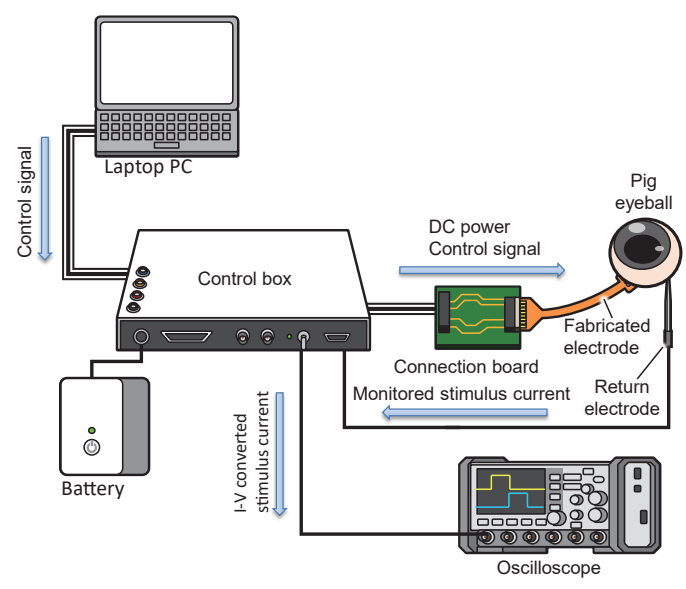

Fig. 14. (Color online) Experimental setup of the ex vivo experiment.
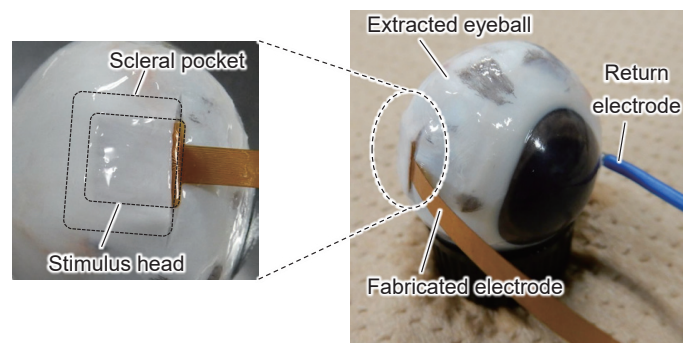

Fig. 15. (Color online) Photograph of extracted pig eyeball after implantation of electrodes. Left photograph shows enlargement of electrode implantation area.

Figure 16 shows the observed waveforms of the stimulus current. The fabricated electrode successfully output a cathodic-first biphasic pulse that was the same as the stimulation used in the clinical trial. ${ }^{(1)}$ The stimulus intensity could be controlled to be the same as the programed value. From these results, the stimulus function of the fabricated electrode was demonstrated successfully under the same implantation situation as in practical use. Although the biphasic pulses were successfully output, the waveform of the stimulus pulse was not rectangular. This is because the stimulus current generator that is integrated into the CMOS microchip was not capable of providing sufficient constant current regulation. The load estimated in the stimulation during the implantation was large in comparison with the validation in the dry state described in Sect. 3.1. The introduction of a circuit with a higher output impedance as an output stage of the stimulus current generator will provide sufficient current regulation capability in our future work. 


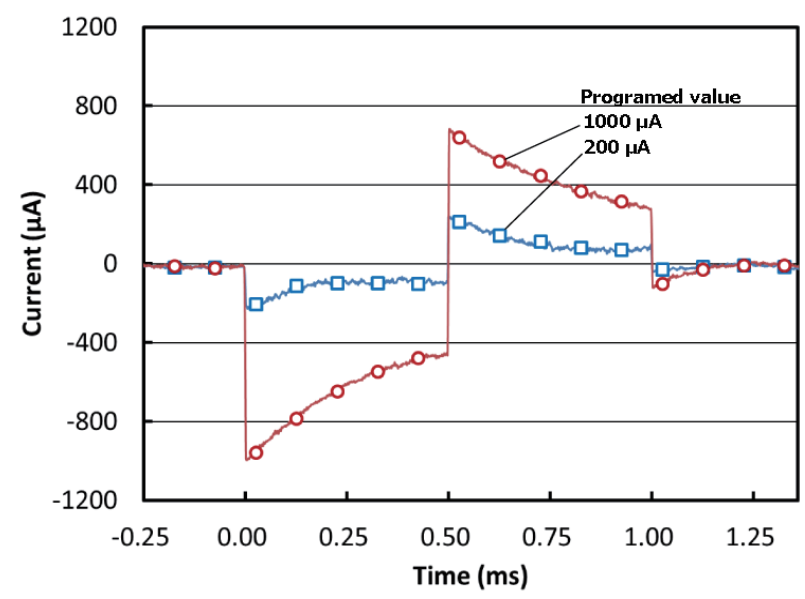

Fig. 16. (Color online) Waveforms of the stimulus current in the ex vivo experiment.

\section{Conclusions}

A smart electrode that has a built-in CMOS microchip was fabricated and demonstrated, with an eye to a next-generation retinal prosthesis. The stimulus function of the fabricated electrode was successfully validated through an ex vivo experiment with the same configuration as that in its potential practical use. From the results of our previous work, ${ }^{(9,10)}$ where we used electrodes of the same size, the threshold current to evoke a retinal reaction is known to be 100-200 $\mu \mathrm{A}$. Because the fabricated electrode demonstrated a sufficient margin of stimulus intensity, retinal cells might be stimulated using the electrode. We plan to perform in vivo experiments to prove the potential of the electrode. In this work, we only demonstrated single-electrode operation to validate the concept. The architecture of the fabricated electrode is suitable for a larger array, which is expected as a next step. The large array may enable high-resolution sight restoration, which will allow for character recognition, and improve the quality of life of patients.

\section{Acknowledgments}

The authors would like to thank the staff of the Vision Institute of NIDEK Co., Ltd., Japan, for technical discussions. This work was partially supported by the General R\&D Subsidies Program from Terumo Life Science Foundation, Japan, and a research grant from Murata Science Foundation, Japan. Design of the microchip was supported by the VLSI Design and Education Center (VDEC), The University of Tokyo, in collaboration with Cadence Corporation.

\section{References}

1 L. Yue, J. D. Weiland, B. Roska, and M. S. Humayun: Prog. Retin. Eye Res. 53 (2016) 21.

2 L. da Cruz, B. F. Coley, J. Dorn, F. Merlini, E. Filley, P. Christopher, F. K. Chen, V. Wuyyuru, J. Sahel, P. Stanga, M. Humayun, R. J. Greenberg, and G. Dagnelie: Br. J. Ophthalmol. 97 (2013) 632. 
3 A. K. Ahuja and M. R. Behrend: Prog. Retin. Eye Res. 36 (2013) 1.

4 Y. H.-L. Luo and L. da Cruz: Prog. Retin. Eye Res. 50 (2016) 89.

5 E. Zrenner, K. U. Bartz-Schmidt, H. Benav, D. Besch, A. Bruckmann, V. P. Gabel, F. Gekeler, U. Greppmaier, A. Harscher, S. Kibbel, J. Koch, A. Kusnyerik, T. Peters, K. Stingl, H. Sachs, A. Stett, P. Szurman, B. Wilhelm, and R. Wilke: Proc. R. Soc. B Biol. Sci. 278 (2010) 1489.

6 V. B. D. Kitiratschky, K. Stingl, B. Wilhelm, T. Peters, D. Besch, H. Sachs, F. Gekeler, K. U. Bartz-Schmidt, and E. Zrenner: Graefes Arch. Clin. Exp. Ophthalmol. 253 (2015) 381.

7 K. Stingl, K. U. Bartz-Schmidt, D. Besch, C. K. Chee, C. L. Cottriall, F. Gekeler, M. Groppe, T. L. Jackson, R. E. MacLaren, A. Koitschev, A. Kusnyerik, J. Neffendorf, J. Nemeth, M. A. N. Naeem, T. Peters, J. D. Ramsden, H. Sachs, A. Simpson, M. S. Singh, B. Wilhelm, D. Wong, and E. Zrenner: Vision Res. 111 (2015) 149.

8 H. Kanda, T. Morimoto, T. Fujikado, Y. Tano, Y. Fukuda, and H. Sawai: Invest. Ophthalmol. Vis. Sci. 45 (2004) 560.

9 T. Noda, K. Sasagawa, T. Tokuda, Y. Terasawa, H. Tashiro, H. Kanda, T. Fujikado, and J. Ohta: Sens. Actuators, A 211 (2014) 27.

10 T. Noda, K. Sasagawa, T. Tokuda, K. Hiroyuki, T. Yasuo, T. Hiroyuki, F. Takashi, and O. Jun: Sens. Mater. 26 (2014) 637.

11 T. Fujikado, M. Kamei, H. Sakaguchi, H. Kanda, T. Morimoto, Y. Ikuno, K. Nishida, H. Kishima, T. Maruo, K. Konoma, M. Ozawa, and K. Nishida: Invest. Ophthalmol. Vis. Sci. 52 (2011) 4726.

12 T. Noda, K. Sasagawa, T. Tokuda, Y. Terasawa, H. Tashiro, H. Kanda, T. Fujikado, and J. Ohta: Electron. Lett. 48 (2012) 1328.

13 T. Noda, T. Fujisawa, R. Kawasaki, H. Tashiro, H. Takehara, K. Sasagawa, T. Tokuda, and J. Ohta: Proc. Annu. Int. Conf. IEEE Engineering in Medicine and Biology Society EMBS 2015 (IEEE, 2015) 3355.

14 Y. Terasawa, H. Tashiro, Y. Nakano, T. Tokuda, and J. Ohta: Adv. Biomed. Eng. 5 (2016) 137. 